\title{
Osteonecrosis of the jaws and non-malignant disease
}

\section{A. Coudert ${ }^{1}$, G. Lescaille, $V^{2}$. Baaroun ${ }^{1,2}$, J. Azérad ${ }^{2}$, M. Cohen-Solal ${ }^{3}$, A. Berdal ${ }^{1}$, V. Descroix ${ }^{1,2}$}

${ }^{1}$ Laboratoire de Physiopathologie Orale Moléculaire - Equipe 5 - Centre de Recherche des Cordeliers - UMRS872 - Paris; ${ }^{2}$ Oral Surgery Department, PitiéSalpêtrière University Hospital, Paris Diderot University, Paris, France ${ }^{3}$ Laboratoire Os et Articulations - Hôpital Lariboisière - UMRS 606 - Paris

\section{Introduction}

Bisphosphonates (BP) are powerful bone resorption inhibitors. They are used for the symptomatic treatment of malignant osteolytic bone disease (e.g., multiple myeloma and bone metastasis), as well as bone diseases associated with high bone resorption (e.g., postmenopausal osteoporosis, cortisone-induced osteoporosis). However, recent data showed that a rare, but serious, adverse effect of BP therapy is bisphosphonate related osteonecrosis of the jaw (BRONJ). Given the increasing number of persons receiving chronic oral-BP therapy, it is important to accurately identify pathogenesis, risk factors and management strategies for BRONJ in patients with non-malignant disease. The objective of this study was to review cases of BRONJ occurring in association with benign disease and to describe and compare the clinical course and outcome for patients with BRONJ and rheumatoid arthritis or osteoporosis.

We retrospectively reviewed observations of all patients referred for treatment and follow-up for BRONJ from January 2007 to December 2011. Demographic data, medical history, maxillofacial findings, BRONJ treatment and follow-up were reviewed for each case. Over a five-year period, we diagnosed 112 patients with BRONJ. Among these patients, 15 received bisphosphonate treatment for non-malignant disease.

\begin{tabular}{|c|c|c|c|c|c|c|c|c|c|c|c|}
\hline Patient & Age (years) & Sex & Disease & Trigerevent & BRONJ site & $\begin{array}{c}\text { BRONJ } \\
\text { stage }\end{array}$ & Bisphosphonate & $\begin{array}{c}\text { Duration (mo) } \\
\text { Cumulative dose (mg) }\end{array}$ & $\begin{array}{c}\text { BRONJ } \\
\text { management }\end{array}$ & $\begin{array}{c}\text { Followup } \\
(\text { mo })\end{array}$ & Comorbidities \\
\hline 1 & 51 & $\mathrm{~F}$ & $\begin{array}{c}\text { Rheumatoid } \\
\text { arthritis }\end{array}$ & Tooth extraction & $\begin{array}{l}\text { Right and left } \\
\text { mandibles }\end{array}$ & 2 & Risedronate & $\begin{array}{c}\mathbf{2 4} \\
3360 \\
\end{array}$ & Medical & Not controlled & $\begin{array}{l}\text { Sjogren's Syndrome } \\
\text { Methotrexate }\end{array}$ \\
\hline 2 & 59 & $\mathrm{~F}$ & $\begin{array}{l}\text { Rheumatoid } \\
\text { arthritis }\end{array}$ & Tooth extraction & Mandible & 2 & Risedronate & $\begin{array}{c}\mathbf{6 0} \\
8400\end{array}$ & $\begin{array}{l}\text { Medical } \\
\text { Surgery }\end{array}$ & $\begin{array}{l}\text { Complete healing } \\
\text { (24) }\end{array}$ & $\begin{array}{c}\text { Hypertension } \\
\text { Phlebitis } \\
\text { Leflunomide } \\
\text { Prednisone } \\
\text { Fluindione } \\
\end{array}$ \\
\hline 3 & 56 & M & $\begin{array}{l}\text { Rheumatoid } \\
\text { arthritis }\end{array}$ & Tooth extraction & Left mandible & 2 & $\begin{array}{l}\text { Zoledronate } \\
\text { Pamidronate }\end{array}$ & $\begin{array}{l}\mathbf{3 6} \\
48 \\
\mathbf{1 2} \\
180 \\
\end{array}$ & Medical & $\begin{array}{l}\text { Complete healing } \\
\text { (6) }\end{array}$ & $\begin{array}{l}\text { Arrhythmias } \\
\text { Fluindione } \\
\text { Bisoprolol } \\
\text { Prednisone } \\
\end{array}$ \\
\hline 4 & 57 & $\mathrm{~F}$ & $\begin{array}{c}\text { Rheumatoid } \\
\text { arthritis }\end{array}$ & Spontaneous & Right maxillar & 1 & Alendronate & $\begin{array}{c}\mathbf{9 6} \\
26880 \\
\end{array}$ & $\begin{array}{l}\text { Medical } \\
\text { Surgery }\end{array}$ & $\begin{array}{l}\text { Complete healing } \\
\text { (3) }\end{array}$ & Adalimumab \\
\hline 5 & 39 & M & $\begin{array}{l}\text { Rheumatoid } \\
\text { arthritis }\end{array}$ & Tooth extraction & $\begin{array}{l}\text { Right and left } \\
\text { mandibles }\end{array}$ & 2 & Alendronate & $\begin{array}{c}\mathbf{3 6} \\
10080\end{array}$ & $\begin{array}{l}\text { Medical } \\
\text { surgery }\end{array}$ & Not controlled & \begin{tabular}{|c|} 
RenalInsufficiency \\
Hydroxychloroquine \\
Methotrexate \\
Prednisone \\
\end{tabular} \\
\hline 6 & 82 & $\mathrm{~F}$ & $\begin{array}{l}\text { Rheumatoid } \\
\text { arthritis }\end{array}$ & Tooth extraction & Mandibular fracture & 3 & $\begin{array}{l}\text { Alendronate } \\
\text { Ibandronate }\end{array}$ & $\begin{array}{c}\mathbf{1 2} \\
280 \\
\mathbf{1 8} \\
2700 \\
\end{array}$ & $\begin{array}{l}\text { Medical } \\
\text { Surgery }\end{array}$ & $\begin{array}{l}\text { Complete healing } \\
\text { (13) }\end{array}$ & $\begin{array}{c}\begin{array}{c}\text { Angor pontage coronarien } \\
\text { stent triatec plavix vastarel } \\
\text { lasilix tenormine lipanthyl } \\
\text { cortancyl }\end{array} \\
\end{array}$ \\
\hline 7 & 84 & $\mathrm{~F}$ & $\begin{array}{c}\text { Rheumatoid } \\
\text { arthritis }\end{array}$ & Tooth extraction & Maxillar sinus & 3 & Ibandronate & $\begin{array}{c}36 \\
5400\end{array}$ & $\begin{array}{l}\text { Medical } \\
\text { Surgery } \\
\end{array}$ & $\begin{array}{c}\text { Complete healing } \\
\text { (24) }\end{array}$ & $\begin{array}{c}\text { Prednisone } \\
\text { Methotrexate } \\
\end{array}$ \\
\hline 8 & 89 & $\mathrm{~F}$ & $\begin{array}{c}\text { Rheumatoid } \\
\text { arthritis }\end{array}$ & Tooth extraction & Right maxillar & 2 & Alendronate & $\begin{array}{c}60 \\
16800\end{array}$ & Medical & $\begin{array}{l}\text { Complete healing } \\
\text { (5) }\end{array}$ & Prednisone \\
\hline 9 & 78 & $\mathrm{~F}$ & Osteoporosis & Tooth extraction & Left mandible & 2 & Risedronate & $\begin{array}{c}36 \\
5400 \\
\end{array}$ & $\begin{array}{l}\text { Medical } \\
\text { Surgery }\end{array}$ & $\begin{array}{c}\text { Complete healing } \\
(6)\end{array}$ & None \\
\hline 10 & 80 & M & Osteoporosis & Tooth extraction & Left mandible & 2 & Ibandronate & $\begin{array}{c}18 \\
2700\end{array}$ & Medical Surgery & $\begin{array}{l}\text { Complete healing } \\
\text { (36) }\end{array}$ & $\begin{array}{c}\text { Diabetes } \\
\text { Parkinson disease } \\
\text { Metformine } \\
\end{array}$ \\
\hline 11 & 79 & $\mathrm{~F}$ & Osteoporosis & Tooth extraction & Right mandible & 2 & Alendronate & $\begin{array}{c}48 \\
13400\end{array}$ & $\begin{array}{l}\text { Medical } \\
\text { Surgery }\end{array}$ & $\begin{array}{l}\text { Complete healing } \\
\text { (3) }\end{array}$ & None \\
\hline 12 & 89 & $\mathrm{~F}$ & Osteoporosis & Tooth extraction & Right maxillar & 2 & Alendronate & $\begin{array}{c}84 \\
23520 \\
\end{array}$ & $\begin{array}{l}\text { Medical } \\
\text { Surgery }\end{array}$ & $\begin{array}{c}\text { Complete healing } \\
\text { (12) }\end{array}$ & None \\
\hline 13 & 70 & $\mathrm{~F}$ & Osteoporosis & Tooth extraction & Left maxillar & 2 & $\begin{array}{l}\text { Alendronate } \\
\text { Risedronate }\end{array}$ & $\begin{array}{c}\mathbf{3 6} \\
10080 \\
\mathbf{7 2} \\
10800 \\
\end{array}$ & Medical & $\begin{array}{c}\text { Complete healing } \\
\text { (8) }\end{array}$ & None \\
\hline 14 & 69 & $\mathrm{~F}$ & Osteoporosis & Tooth extraction & Left maxillar & 2 & Alendronate & $\begin{array}{c}36 \\
10080 \\
\end{array}$ & Medical & $\begin{array}{c}\text { Complete healing } \\
\text { (4) }\end{array}$ & None \\
\hline 15 & 64 & $\mathrm{~F}$ & Algodystrophy & Spontaneous & $\begin{array}{c}\text { dental implant right } \\
\text { mandible }\end{array}$ & 2 & Zoledronate & $\begin{array}{l}6 \\
5 \\
\end{array}$ & $\begin{array}{l}\text { Medical } \\
\text { Surgery }\end{array}$ & $\begin{array}{l}\text { Complete healing } \\
(6)\end{array}$ & Diclofenac omeprazole \\
\hline
\end{tabular}

Table 1: Patient characteristics $(n=15)$. BRONJ was diagnosed following tooth extraction except in Patients 4 and 15, whose diagnoses were spontaneous.

Medical treatment: oral rinse and oral antibiotics (amoxicillin $1 \mathrm{~g}$ plus clavulanic acid $125 \mathrm{mg}$ po twice daily or clindamycin $600 \mathrm{mg}$ po plus metronidazole $500 \mathrm{mg}$ po twice daily). BRONJ: bisphosphonate-related osteonecrosis of the jaw; BP: bisphosphonate; RA: rheumatoid arthritis; SS: Sjögren syndrome; MTX: methotrexate; HTN: hypertension; LEF: leflunomide; HCQ: hydroxychloroquine; CAD: coronary artery disease OP: osteoporosis; AD: algodystrophy.

\begin{tabular}{|c|l|}
\hline $\begin{array}{l}\text { At risk } \\
\text { category }\end{array}$ & $\begin{array}{l}\text { No apparent exposed/necrotic bone in patients who have been treated with either oral or IV } \\
\text { bisphosphonates }\end{array}$ \\
\hline Stage $\mathbf{0}$ & $\begin{array}{l}\text { Nonspecific clinical findings and symptoms such as jaw pain or osteosclerosis but no clinical } \\
\text { evidence of exposed bone }\end{array}$ \\
\hline Stage $\mathbf{1}$ & Exposed/necrotic bone in patients who are asymptomatic and have no evidence of infection. \\
\hline Stage $\mathbf{2}$ & $\begin{array}{l}\text { Exposed/necrotic bone associated with infection as evidenced by pain and erythema in the region } \\
\text { of the exposed bone with or without purulent drainage }\end{array}$ \\
\hline Stage 3 & $\begin{array}{l}\text { Exposed/necrotic bone in patients with pain, infection, and one or more of the following: } \\
\text { pathologic fracture, extra-oral fistula, or osteolysis extending to the inferior border or sinus floor }\end{array}$ \\
\hline
\end{tabular}

Table 2: BRONJ patients were characterized according to the the AAMOS guidelines (Ruggeriro SL, 2009)
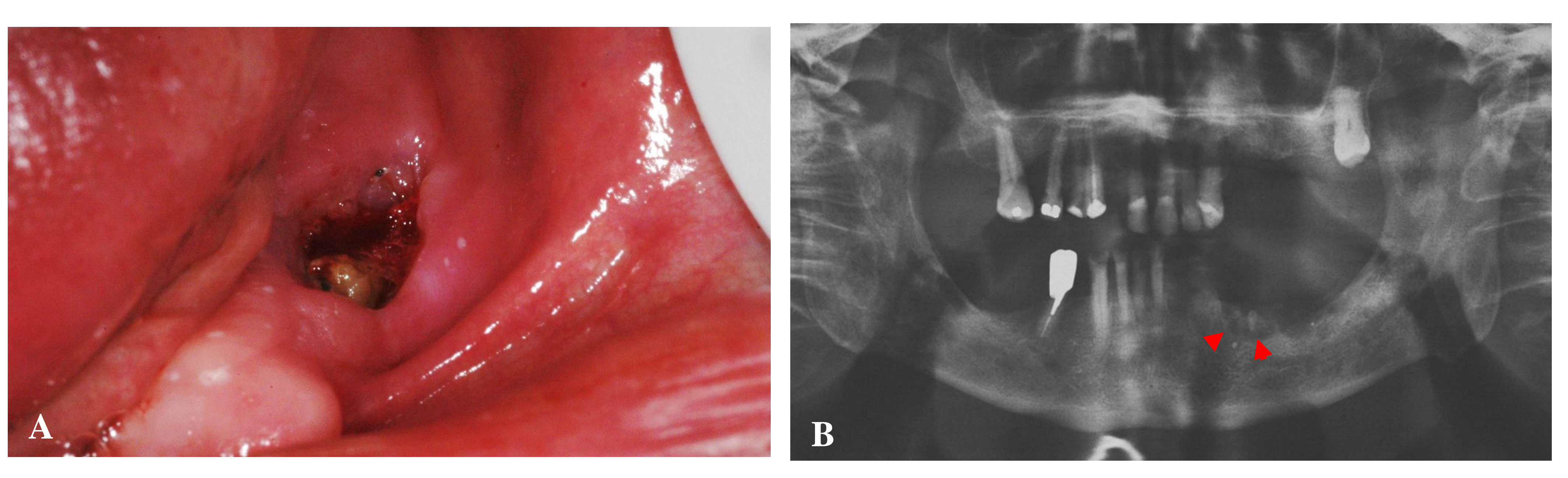

Figure 1: Typical stage 2 BRONJ. A. Clinical photograph showing exposed bone following a tooth extraction. B. Panoramic X-ray showing the lost of mandibular bone and the characteristic radioluscent and radio opaque lesion (red arrow heads).

\begin{tabular}{|c|c|c|c|}
\hline Criteria & $\mathbf{R A}(\mathbf{n}=\mathbf{8})$ & Others (n=7) & $\boldsymbol{p}$ \\
\hline Age (mean, years) & 64.63 & 75.57 & $n s$ \\
\hline Female (\%) & $75 \%$ & $85 \%$ & $n s$ \\
\hline BP duration (mean, months) & 48.75 & 48 & $n s$ \\
\hline Glucocorticoids & $75 \%$ & $0 \%$ & \\
\hline DMARDs & $65.2 \%$ & $0 \%$ & \\
\hline Alendronate (mean, mg) & 13510 & 14270 & $n s$ \\
\hline Trigger event (extraction) & $87.5 \%$ & $85.7 \%$ & $n s$ \\
\hline BRONJ stage & & & $n s$ \\
\hline $\begin{array}{c}\text { Stage 1 (\%) } \\
\text { Stage 2 (\%) }\end{array}$ & $12.5 \%$ & $0 \%$ & $n s$ \\
\hline Stage 3 (\%) & $25 \%$ & $100 \%$ & $n s$ \\
\hline Surgery & $62.5 \%$ & $71.4 \%$ & $n s$ \\
\hline $\begin{array}{c}\text { Complete healing (mean, } \\
\text { months) }\end{array}$ & 12.5 & 10.71 & $n s$ \\
\hline
\end{tabular}

Table 3. Comparison of clinical BRONJ criteria between patients with rheumatoid arthritis (RA) and those with other nonmalignant disease. Data are $n(\%)$ unless otherwise indicated. (RA) and those with other nonmalignant disease. Data are $\mathrm{n}(\%)$ unless otherwise indicated.
$\mathrm{p}$ values were not significant. DMARDs, disease-modifying antirheumatic drugs : methotrexate, leflunomide, adalimumab, hydroxychloroquine,

\section{Conclusion}

Comparative analysis of all the variables showed no statistically significant differences between patients with rheumatoid arthritis and others. However, our study is the first, to our knowledge, to distinguish and compare 2 groups of patients treated with BP for non malignant disease. In conclusion, within the limits of our study (small size cohort, lack of control group, patients referred to our department for ONJ), our results suggest that RA does not alter the BRONJ disease spectrum, clinical course or outcome. Further studies are needed to assess the incidence and prevalence of osteonecrosis of the jaws in patients with RA. 